\title{
Perspectives From Working Parents of Children With Recurrent Acute Otitis Media During The COVID-19 Era: A Qualitative Study
}

Alia Badawi ( $\square$ abadawi1@jh.edu )

Johns Hopkins University

Christine Santos

Johns Hopkins University

Keith Rochkind

Johns Hopkins University

James H. Clark

Johns Hopkins University

Therese L. Canares

Johns Hopkins University

\section{Research Article}

Keywords: Otitis Media, Qualitative Research, Pediatrics, Parents, COVID-19, Sars-CoV-2, Work-Life Balance, Working Women

Posted Date: March 10th, 2021

DOl: https://doi.org/10.21203/rs.3.rs-228855/v1

License: (c) (1) This work is licensed under a Creative Commons Attribution 4.0 International License. Read Full License 


\section{Abstract}

\section{Background}

Acute otitis media (AOM) in children is a common pediatric illness which can cause significant pain and discomfort. For families of children with recurrent AOM there are additional burdens to consider, including but not limited to lost workdays, direct costs for medical care and follow up treatments, and indirect costs like childcare and transportation. With the emergence of the COVID-19 pandemic, parents also have to contend with the added burden of balancing their child's needs to seek medical care and the risk of exposure to COVID-19. The aim of this study was to characterize working parents' experiences around their children's ear infections during the COVID-19 era.

\section{Methods}

This was a qualitative study based on semi-structured interviews with working parents of children with recurrent ear infections. Using grounded theory methods, content analysis was applied to identify themes that described the parental experience of AOM during the COVID-19 pandemic.

\section{Results}

Eleven parents were interviewed. Notable themes were: parents described a lack of confidence in their children's AOM diagnosis or treatment; frequent doctor's visits for quick middle ear checks were inconvenient and unsatisfactory, and this was exacerbated by both COVID-19 and pressures from worklife demands; and parents described a desire for more diagnostic information at home and remote capability for ear infection telehealth check-ups.

\section{Discussion}

This study identified working parents' challenges and dissatisfaction with taking their child to a doctor and their desire for alternative screening methods for their child's ear infections at home, particularly during the COVID-19 pandemic. Clinicians might address working parents' needs with validation of their difficulties, while collaborating with parents on novel approaches to remote monitoring of ear health.

\section{Introduction}

Acute otitis media (AOM) in children is a common pediatric illness which can cause significant pain and discomfort. Global annual incidence among 1-4-year-olds is reported to be as high as $60.99 \%$.[1] While the physical burdens of AOM can be pronounced for children, for young children in particular, the impact on language, cognitive, and social development can also be significant and potentially impact long-term achievement. For families of children with recurrent AOM there are additional burdens to consider, including but not limited to lost workdays, increased direct costs for medical care and follow up treatments, and increased indirect costs like childcare and transportation.[2] Historically and in present times, mothers take on a disproportionate burden of childcare responsibilities, which is exacerbated 
during a child's acute illness.[3, 4] Beyond these health and economic burdens, parents caring for children with AOM may also experience increased feelings of anxiety and guilt, commensurate with the level of disruption to the normal routine of family life. With the emergence of the COVID-19 pandemic, parents now also have to contend with the added challenge of balancing their child's needs to seek medical care and the risk of exposure to COVID-19 upon visiting a healthcare facility.[5]

It is unclear how the COVID-19 pandemic has affected access to and utilization of healthcare for children with ear disease. At a larger scale there have been significant drops, up to $42 \%$, for pediatric emergency department (ED) and primary care visits when compared to similar periods in years prior since the COVID19 pandemic began.[5-9] Some countries have reported lower numbers of ED visits for AOM, but it is hard to decipher whether this trend is the product of reduced social contact and decreased transmission risk for virus related $\mathrm{AOM}$ or due to delayed care seeking by parents concerned about the risk of exposure to COVID-19.[10, 11]

The health system must evolve to meet patients' needs in light of a pandemic where patients may have reduced access to care, and families must contend with a new type of work-life balance. Currently there is no data that describes the impact of COVID-19 on the pediatric patient and family experience of AOM. With increasing utilization of telehealth there is also a need to better understand the difficulties and concerns parents confront when considering telemedicine for illnesses like AOM.[12-14] The aim of this study is to characterize working parents' experiences around their children's ear infections during the COVID-19 era.

\section{Methods}

\section{Study Design}

This was a qualitative study using grounded theory methodology that entailed interviewing working professional parents of children with recurrent ear infections from June-July 2020.

\section{Study Setting \& Population}

All interviews took place through phone call or video conference call, in order to maintain physical distancing guidelines during the COVID-19 pandemic. A cohort of working professional parents, specifically mothers, was purposefully sampled to characterize the intersect of family and career, and with the theory that this subset would be particularly burdened by the impact of COVID-19 on their children's healthcare issues. This cohort was identified through social media advertisements on parent groups, seeking working professional mothers whose children have had recurrent ear infections or required tympanostomy tube surgery. Exclusion criterion was non-English speakers.

\section{Data Collection and Analysis}

The Johns Hopkins University School of Medicine Institutional Review Board (IRB) approved this study; informed consent was obtained. This study was performed in accordance with the Johns Hopkins 
University School of Medicine IRB ethical guidelines on human subjects research.

To develop an understanding of working parents' experiences on their child's ear infections during the COVID-19 era, we used grounded theory methodology to identify themes on this topic.[15] The senior investigator [TC] who is trained in qualitative research and has published research using qualitative methodologies guided the study design and trained the investigative team. A semi-structured interview guide was developed by the senior investigators [JC, TC], and included probing questions on emotions, their needs during a medical encounter, professional issues, and the impact of COVID-19. The senior investigators $[T C, J C]$ along with primary investigators $[A B, C S]$ conducted all semi-structured interviews.

Interview audio was digitally recorded, transcribed with Otter.ai software (Otter.ai. Version 2.1.41.612. Lost Altos, CA: Otter.ai; 2020). and final edits made by the research team. The primary outcomes were themes that characterized the participants' experiences.

The coding scheme was drafted by the senior investigator [TC] and edited through consensus with the research team. Initial coding of interview transcripts was performed by two randomly assigned independent raters on the research team $[A B, K R, J C, T C]$, and proceeded iteratively. As additional interviews were conducted, investigators $[A B, T C]$ performed intermediate coding and comparative analysis to identify themes and sub-themes within the coded data. Discrepancies in codes or themes were resolved by group consensus. The process of interviews, coding, debriefs, and thematic analysis proceeded iteratively. For any new themes that emerged the interview guide was adapted to explore those themes in subsequent interviews. Analysis was performed manually, without software tools.

Interviews proceeded until data saturation was reached, i.e. no new codes or themes were identified. (Appendix 1. Interview guide)

\section{Results}

Eleven mothers were interviewed. Demographic data are noted in Table 1. The mean age (standard deviation) was $38( \pm 2.9)$. The median (interquartile range) number of children was $2(0.5)$. Most had advanced degrees (82\% had graduate degrees), and all were employed. Data saturation occurred after 6 interviews.

\section{Theme 1}

\section{Parents' general challenges were related to provider distrust due to diagnostic error, recurrent antibiotic use, and low perceived value of the AOM diagnostic process.}

When asked about challenges related to their children's ear infections, parents expressed frustration and distrust related to medical management, and concern about recurrent antibiotic use. Several parents described visiting multiple providers during the course of an illness and receiving inconsistent diagnoses from one visit to the next. This became particularly burdensome when a changed diagnosis resulted in a perceived delay in antibiotics. Parents also noted variability in accuracy of ear infection diagnoses, 
depending on the type of medical provider. They felt more distrustful of advanced practice providers (nurse practitioners, physician assistants) with less training than seasoned physicians or distrustful of primary care providers over otolaryngology specialists. Another concern was related to the ramifications of frequent antibiotic use while lacking faith in the diagnostic process.

Parents further expressed a helplessness around medical-decision making as parents felt reliant on a provider's imperceptible and sometimes inconsistent observations on the ear exam. The need to rely on a provider's ear exam exacerbated skepticism in the treatment process because the findings on the ear exam were inappreciable for the parent. In general, decreased confidence in the medical management of AOM, stress associated with caring for a sick child, and the effort or inconvenience of a healthcare visit was associated with parents' perceived low value of visits. Despite a perception of low value, parents acknowledged the reliance on their medical team to support care for their child's AOM episodes. (Table 2)

Table 2. Theme 1 Illustrative Quotes. Theme: Parents' general challenges were related to provider distrust due to diagnostic error, recurrent antibiotic use, and low perceived value of the AOM diagnostic process.

\section{Subthemes Quote}

Distrust in "So when he was first diagnosed with his serous otitis [media] he was getting different providers of varying levels deaf. And the pediatrics resident who examined him said that his ears were fully normal. And then the day later the ENT looked at his ears and he said that's not normal at all."

"If I get a nurse practitioner or somebody who's not the physician then I bet I know that there's data to say that there's more variability in [the AOM] diagnosis."

Frustration at treatment delays or diagnostic error
"I was really irritated that our doctor didn't send us [to ENT] sooner because I'm like she was on all those antibiotics like it killed her frickin gut bacteria and Lord knows what else to her, not healthy you know..."

"I could tell she had issues and it would take four days for them to actually, you know, [diagnose her]. I take her and they're like, she's fine she's teething...that's [not an ear infection] ... Then she would get to the point she was screaming. I take her back and then [the doctors] say oh yeah she has an ear infection and like I told you she had an ear infection when we first came in....I've actually ended up switching pediatricians."
Stress around antibiotic overuse

\section{"Getting antibiotics that much was stressful."}

"Systemically having to deal with antibiotics, especially in a baby so young, it just kills me."

"They [doctor's office] said, 'Oh it's ear infections or here are some antibiotics, have a great day.' Yeah. So there wasn't really much they could offer on their end either."

Value of visit: large hassle for a "quick peek"
"Sometimes I would go [to the doctor] and they wouldn't have the ear infection. And it was like all this wasted time and energy."

"[I really wish] I didn't have to schlep him into the doctor's office, just for that two second peek in the ear." 


\section{Theme 2}

\section{The COVID-19 pandemic exacerbated the stress and difficulty of arranging healthcare visits for ear- related issues.}

The COVID-19 pandemic exacerbated parents' aversion to frequent, in-person medical visits for their child's recurrent AOM or tympanostomy tubes. Several parents echoed that, pre-pandemic, the decision of when to bring their child in for AOM suspicion was nebulous due to unclear and abstruse symptoms. That uncertainty on whether to seek medical care was compounded by the COVID-19 pandemic. COVID19 exposure from waiting rooms with potentially infectious patients weighed heavily against a lack of clear AOM indicators when deciding whether to bring their children in for in-person evaluations. The risk of COVID-19 exposure for in-person visits also caused parents to question the necessity of routine visits or, for those with tympanostomy tubes, follow-up visits for the tubes when asymptomatic. Both parents and providers executed this risk-benefit analysis for in-person visits which oftentimes resulted in appointment cancellations and procedure delays, such as a scheduled tympanostomy tube removal. Several parents highlighted that the inability for their physicians to examine AOM remotely meant that AOM-related care was entirely inaccessible when the risk of COVID-19 exposure was unacceptably high. (Table 3)

Table 3: Theme 2 Illustrative Quotes. Theme: The COVID-19 pandemic exacerbated the stress and difficulty of arranging healthcare visits for ear-related issues.

\begin{tabular}{|c|c|}
\hline Subthemes & Quote \\
\hline \multirow{2}{*}{$\begin{array}{l}\text { The risk of } \\
\text { COVID } \\
\text { exposures } \\
\text { for in- } \\
\text { person } \\
\text { visits }\end{array}$} & $\begin{array}{l}\text { "COVID has changed that for me, before we would take our kids to the doctor but } \\
\text { now there is like the added burden of exposure." }\end{array}$ \\
\hline & $\begin{array}{l}\text { "Our pediatrician's office has been pretty spot on, and I'm agreeing with their } \\
\text { [personal protective equipment] protocols and virtual waiting rooms. But... [because of } \\
\text { COVID] if for any reason we had to take our kids into the [emergency room] ... it will } \\
\text { cause me [to] pause before we go to really try to determine, you know, if that's what we } \\
\text { need to do." }\end{array}$ \\
\hline \multirow{2}{*}{$\begin{array}{l}\text { Procedural } \\
\text { delays due } \\
\text { to COVID }\end{array}$} & $\begin{array}{l}\text { "He was supposed to have [tubes] removed this spring but he did not because of } \\
\text { COVID." }\end{array}$ \\
\hline & $\begin{array}{l}\text { "[The ENT] gave us the option ...she could do an outpatient surgery... she would } \\
\text { have been sedated [and] they take the tubes out... then they put a little patch graft, so } \\
\text { they know it's closed and you ... don't have to wait it out. And with the pandemic I'm } \\
\text { like, forget it like the kids are home... I can't coordinate ... taking her to, you know, the } \\
\text { surgery center and like then also waiting for... a COVID test and I'm like, forget it, we'll } \\
\text { just do it in the office. Once the office... open[s] back up" }\end{array}$ \\
\hline
\end{tabular}

\section{Theme 3}


Working parents experienced stress, guilt, and judgement during professional absences related to their child's recurrent AOM.

These working parents described that balancing their professional and family lives during their child's frequent AOMs was a tremendous source of stress. The work-related burdens were related to disrupted productivity, feeling judged or unsupported by peers or superiors, and the logistics of planning a work absence and a medical visit at the same time. Parents who were in a relatively new position or junior role described added worries over negative performance evaluations in light of family medical leave. Feelings of regret and guilt characterized this bidirectional pull between professional and family lives. The stress, regret, guilt, and perceived criticism from work were most strongly felt by parents with frequent, consecutive AOM visits. (Table 4)

Table 4. Theme 3 Illustrative Quotes. Theme: Working parents experienced stress, guilt, and judgement during professional absences related to their child's recurrent AOM.

\begin{tabular}{|c|c|}
\hline Subthemes & Quotes \\
\hline \multirow{2}{*}{$\begin{array}{l}\text { Frequent } \\
\text { time off } \\
\text { caused worry } \\
\text { about work } \\
\text { performance } \\
\text { evaluations }\end{array}$} & $\begin{array}{l}\text { "I felt like [I was] under a microscope and I felt like sometimes my performance } \\
\text { as an employee would be evaluated based on like just attending to my kids medical } \\
\text { needs in general" }\end{array}$ \\
\hline & $\begin{array}{l}\text { "I missed a ton of work; it was very stressful. Yeah, I'm not even sure how I like } \\
\text { got through that, to be honest with all the work I missed" }\end{array}$ \\
\hline \multirow{2}{*}{$\begin{array}{l}\text { Stressors } \\
\text { related to } \\
\text { caring for } \\
\text { their family } \\
\text { whilst having } \\
\text { work } \\
\text { obligations }\end{array}$} & $\begin{array}{l}\text { "It was definitely burdensome on our family and just dealing with every single } \\
\text { one of my [three] kids [ear infections] was a challenge." }\end{array}$ \\
\hline & $\begin{array}{l}\text { "I was just like this is crazy...we'd have to take off work and then drive her to } \\
\text { the doctor's, we'd have to call the pediatrician and then hope to get through and hope } \\
\text { that they had an appointment readily available. It was always, because of work, it } \\
\text { was... an emergency to get in there as fast as we could so we could get her on } \\
\text { medication... It was just like this huge hassle every single week." }\end{array}$ \\
\hline
\end{tabular}

\section{Theme 4}

\section{Parents want to feel more empowered in their decisions to seek care or initiate treatment, through more transparent diagnostic information.}

Parents universally expressed a desire to independently understand the status of their child's ear exam. Several parents pointed to their frustration around diagnostic ambiguity and the disruptive nature of frequent, sometimes weekly, AOM-related visits. Many suggested that a home use device would enable direct observation of their child's eardrum with less reliance on medical providers, especially when trust and confidence in their medical team was impaired. Parents imagined a tool that performed a screening exam at home that could help them gauge the necessity of seeking in-person care, a decision with higher stakes during the COVID-19 pandemic. The added value of a home screening would rule out causes with similar presentations to AOM that do not warrant an in-person visit, (e.g. upper respiratory viral infection, 
teething, or tympanostomy tube disruption). Some noted that if such a device could share data with their medical providers, many AOM-related visits could be conducted remotely. In this proposed scenario remote medical evaluations were especially desirable when COVID-19 exposures presented excessive risk. A device with transparent diagnostic information would address the stress around their child's symptom ambiguity and empower parents to make more informed decisions. (Table 5)

Table 5. Theme 4 Illustrative Quotes. Theme: Parents want to feel more empowered in their decisions to seek care or initiate treatment, through more transparent diagnostic information.

\section{Subthemes Quotes} urgency for doctor's visit when symptoms were ambiguous, or patients were pre-verbal

Desire for a quick baseline diagnostic when deciding next steps
Stressful to determine

"Her symptoms were like disrupted sleep, like that was it. She was never feverish, never really fussy. And so disrupted sleep ... can be anything. It can be teething, it can be illness, it can be an ear infection, it could be nothing, like, it [could] just be a Tuesday."

"As a parent I think just the hardest thing is not knowing, my kids just presented very differently."

"If there was a way for me to just like quickly say yes it meets this threshold that we're concerned that it's an ear infection like it would have been so much easier for me to just stay home sometimes and like ride some of these things out and not feel like I was being negligent."

"It would have been really nice if we had some route to monitor that at home as I felt like it was so frustrating for me to have to go into the doctor's office."

Enable ear assessments via Telehealth during COVID-19
"I wonder if ... there was a way to ... send a photo to a doctor so you could just skip the doctor's visit altogether, and only have to go to the pharmacy."

"It would be really disarming to have to constantly go into the pediatricians office [during COVID] ... If I could have something at home that... [could show] the data and send it to [the doctor for] a telehealth medicine visit, that would be incredible."

\section{Discussion}

This qualitative study characterized the experiences of working parents of children with recurrent ear infections during the COVID-19 pandemic. Themes of their experiences related to delayed care due to fear of exposure to COVID-19, low perceived value of AOM-related visits, added burdens of work-life balance during a child's recurrent illnesses, and a desire for information to guide parent decisions.

\section{The Implications of Delayed AOM Care during COVID-19}

Parents in this study identified that COVID-19 led to delays or hesitation about seeking care for their child's ear complaints. Reluctance to seek care for ill children during the COVID-19 pandemic has been observed in other settings.[5-9] Delayed care for a child's AOM, as seen in the present study, can have deleterious effects in rare cases, or may be of minimal consequence in non-severe cases. In severe cases 
mastoiditis is a complication of recurrent AOM[16], and in instances of chronic or complicated otitis media procrastination of surgery increases the risk of intracranial complications.[17] Conversely, the "watchful waiting" approach for non-severe AOM results in satisfactory recovery without antibiotic use and therefore avoids patients unnecessarily developing multi-drug resistance.[18] Since outcomes of untreated $A O M$ range from self-resolving to life threatening intracranial infections, this underscores the importance of clinician involvement to accurately monitor and document the course of AOM. Hesitation for a medical evaluation due to COVID-19, as was observed in our study, may inadvertently lead to more patients utilizing "watchful waiting" for AOM, or conversely antibiotics prescribed via telehealth despite an incomplete ear exam. The impact of COVID-19 on antibiotic usage for AOM has yet to be determined. In the setting of the COVID-19 pandemic and thereafter, clinicians must adapt to supporting patients' concerns for infectious exposures from a medical visit, while minimizing complications of delayed AOM treatment.

\section{Parents Perceived Low Value of Healthcare Visits}

This study found that parents report perceived low value for diagnostic AOM visits when comparing logistical stress with the short visit time. Existing research supports that parents view caring for their child's AOM as a burden.[19-21] Chando et al conducted a systematic review of seventeen studies describing parental experiences of caring for a child with $\mathrm{OM}$ and identified seven central themes including doubt in treatment efficacy and diminishing parental competency that worsen with AOM chronicity. In addition to these previously identified themes of parental experiences, the present study identified a novel aspect of parents' experience in how parents appraise the value of office visits for their child's AOM. Parents perceived that a low-value visit, based on logistical and emotional effort plus trust in providers' diagnostic accuracy, negatively impacted their decision to seek acute care or a bias against particular providers. In an era of value-based care, where patient engagement, patient satisfaction, and quality of care can impact reimbursement, the perceived value of visits by patients is relevant to providers and health systems. Clinicians seeking to maintain healthy relationships with patients and families might incorporate messages of validation to the difficult parental experience around chronic AOM and addressing family needs to maximize value of the visit.

\section{Evolving Desire for Health Information}

Parental distress about medical visits during the COVID-19 pandemic, combined with dissatisfaction with the inexact diagnostic process, and distrust of their provider's recommendations led to parents desiring better information about their child's ear infections. These stressors and challenges led to parents expressing desire for a tool that would facilitate at home diagnosis of AOM or remote tympanostomy tube monitoring. Other studies have also demonstrated information gaps and a desire from parents for more clear information from physicians regarding treatment options for AOM.[22-24] In an effort to seek guidance parents will source information from other sources (internet, friends, family), in conjunction with the information they receive from family physicians, to better inform their understanding and decision making process.[22] Telemedicine is one modality for parents to monitor a child's ear condition. While 
telemedicine use has accelerated during the COVID-19 pandemic[14], challenges remain in the ease of use, accessibility, lack of ubiquity, and integration by physicians.[25] However, as previous studies have shown, parental interest in better information to understand and facilitate the diagnostic and treatment process for childhood illnesses, like AOM, remains strong.[23] This desire, along with the existing usability and lack of universality with at-home otoscopy, warrants both future exploration in telehealth feasibility for AOM and innovation of products that meet these needs.

\section{The Pressures of Work-Life Balance}

The COVID-19 pandemic may have brought to a head the stresses families face balancing the demands of work and family life with meeting the medical needs of children facing common childhood illnesses like AOM. Our study demonstrated that among working professional parents a child's AOM can present a number of work-life challenges. Among these challenges are disruptions to the family routine, personal pressures arising from feelings of guilt and uncertainty, and external pressures from work. Other studies have reported similar emotional and social impacts from AOM on parents, such as disrupted family schedules, parental anxiety, financial strain, etc.[23] Work-life challenges parents face when coping with a child's AOM have also been demonstrated in other studies on the burden of AOM on families.[2, 19, 26] Among parents of children with AOM, $52 \%$ indicated they had to miss some work days, often 2-3 days at a time.[19,26] Consequences of work absence primarily included loss of income and problems at work for parents themselves and their colleagues.[19] For employees lacking well established sick leave policies, the financial consequences of caring for a child with AOM could be much greater on families, especially in cases of recurrent AOM. This study underscores the need for flexibility and support of parents whose children need medical attention, especially when these circumstances are urgent, unplanned, or unexpectedly time consuming.

\section{Limitations}

This study is limited, in part, by generalizability. This study purposively sampled working mothers who, based on past qualitative studies of parental experience of children's AOM [22, 23, 27], are often the primary caregivers for their children's' illnesses. There was also a predominance of mothers with professional degrees or roles. The perspective of this cohort may not characterize the parental experience for fathers or non-binary parents, or parents without advanced degrees or non-professional roles.

Nevertheless, this study offers insight into the parental experience during the unprecedented period of the COVID-19 pandemic. This study may also be limited by response bias, as the parents who enrolled in the study may have had especially evocative experiences that motivated their participation. In addition, this study is subject to recall bias, as some parents described events that occurred before the time of the interviews. Given that the themes identified in this study were experienced across the sampled population and were consistent with past work in this area[21], response and recall bias are less likely to impact the findings of this study. Finally, there is a possibility of experimenter bias, whereby the interviewer can influence the responses by the participants. Measures taken to reduce this were use of a semi-structured 
interview guide, and interviews were conducted by a researcher trained in qualitative research methods whereby prompts were asked as open-ended questions.

\section{Conclusion}

This qualitative study of interviews with working, professional parents of children with recurrent ear infections found that the COVID-19 pandemic has compounded the pre-existing difficulties around caring for an acutely ill child. Parents identified low value in their child's ear-related medical visits and are seeking alternate methods to screen their child at home for ear disease. Pediatric clinicians who care for children with recurrent ear complaints might focus on validating the difficult family experience and can consider leveraging existing technology and telehealth for remote assessments of ear health.

\section{Declarations}

Ethics approval and consent to participate: The Johns Hopkins University School of Medicine Institutional Review Board approved this study protocol. This study was performed in accordance with the Johns Hopkins University School of Medicine IRB ethical guidelines on human subjects research. Informed consent was obtained from all participants.

Consent for publication: Not applicable

Availability of data and materials: The datasets used and/or analyzed during the current study are available from the corresponding author on reasonable request.

Competing interests: The authors $(\mathrm{TC}, \mathrm{JC})$ are currently in the process of patenting a medical device related to the issues discussed in your research (e.g. ear disease), but the authors are not receiving any financial backing related to this medical device to conduct the research described in the present manuscript.

Funding: This work was supported by the Leon Lowenstein Foundation, New York, NY and Johns Hopkins Technology Ventures, Johns Hopkins University, Baltimore, MD.

\section{Author Contributions:}

The authors have made substantial contributions to the content of the manuscript including:

Conceptualization ( $A B, C S, J C, K R, T C)$, Methodology ( $A B, C S, J C, K R, T C)$, Formal Analysis ( $A B, J C, K R$, $T C)$, Investigation ( $A B, C S, J C, K R, T C)$, Resources ( $A B, C S, J C, K R, T C)$, Data Curation ( $A B, C S, J C, K R, T C)$, Interpretation of Analysis ( $A B, C S, J C, T C)$ Writing- Original Draft ( $A B, C S, T C)$, Writing- Review \& Editing $(A B, C S, J C, K R, T C)$, Visualization ( $A B, C S, T C)$, Supervision (JC, TC), Project Administration (AB, CS), Funding Acquisition (JC, TC).

Acknowledgements: None 


\section{References}

1. Kautz-Freimuth S, Redaelli M, Samel C, Civello D, Altin SV, Stock S: Parental views on acute otitis media (AOM) and its therapy in children-results of an exploratory survey in German childcare facilities. BMC Pediatr 2015, 15:199.

2. Greenberg D, Bilenko N, Liss Z, Shagan T, Zamir O, Dagan R: The burden of acute otitis media on the patient and the family. Eur J Pediatr 2003, 162(9):576-581.

3. Starmer AJ, Frintner MP, Matos K, Somberg C, Freed G, Byrne BJ: Gender Discrepancies Related to Pediatrician Work-Life Balance and Household Responsibilities. Pediatrics 2019, 144(4).

4. Lachance-Grzela M, Bouchard G: Why Do Women Do the Lion's Share of Housework? A Decade of Research. Sex Roles 2010, 63(11-12):767-780.

5. Ciacchini B, Tonioli F, Marciano C, Faticato MG, Borali E, Pini Prato A, Felici E: Reluctance to seek pediatric care during the COVID-19 pandemic and the risks of delayed diagnosis. Ital J Pediatr 2020, 46(1):87.

6. Hartnett KP, Kite-Powell A, DeVies J, Coletta MA, Boehmer TK, Adjemian J, Gundlapalli AV, National Syndromic Surveillance Program Community of P: Impact of the COVID-19 Pandemic on Emergency Department Visits - United States, January 1, 2019-May 30, 2020. MMWR Morb Mortal Wkly Rep 2020, 69(23):699-704.

7. Isba R, Edge R, Auerbach M, Cicero MX, Jenner R, Setzer E, Broughton E, Keegan T: COVID-19: Transatlantic Declines in Pediatric Emergency Admissions. Pediatr Emerg Care 2020, 36(11):551553.

8. Isba R, Edge R, Jenner R, Broughton E, Francis N, Butler J: Where have all the children gone? Decreases in paediatric emergency department attendances at the start of the COVID-19 pandemic of 2020. Arch Dis Child 2020, 105(7):704.

9. Lazzerini M, Barbi E, Apicella A, Marchetti F, Cardinale F, Trobia G: Delayed access or provision of care in Italy resulting from fear of COVID-19. Lancet Child Adolesc Health 2020, 4(5):e10-e11.

10. Angoulvant F, Ouldali N, Yang DD, Filser M, Gajdos V, Rybak A, Guedj R, Soussan-Banini V, Basmaci R, Lefevre-Utile A et al: COVID-19 pandemic: Impact caused by school closure and national lockdown on pediatric visits and admissions for viral and non-viral infections, a time series analysis. Clin Infect Dis 2020:ciaa710. Epub ahead of print.

11. Torretta S, Capaccio P, Coro I, Bosis S, Pace ME, Bosi P, Pignataro L, Marchisio P: Incidental lowering of otitis-media complaints in otitis-prone children during COVID-19 pandemic: not all evil comes to hurt. Eur J Pediatr 2020:[Epub ahead of print].

12. Barney A, Buckelew S, Mesheriakova V, Raymond-Flesch M: The COVID-19 Pandemic and Rapid Implementation of Adolescent and Young Adult Telemedicine: Challenges and Opportunities for Innovation. J Adolesc Health 2020, 67(2):164-171.

13. Ramaswamy A, Yu M, Drangsholt S, Ng E, Culligan PJ, Schlegel PN, Hu JC: Patient Satisfaction With Telemedicine During the COVID-19 Pandemic: Retrospective Cohort Study. J Med Internet Res 2020, 
22(9):e20786.

14. Grossman Z, Chodick G, Reingold SM, Chapnick G, Ashkenazi S: The future of telemedicine visits after COVID-19: perceptions of primary care pediatricians. Isr J Health Policy Res 2020, 9(1):53.

15. Chun Tie $Y$, Birks $M$, Francis $K$ : Grounded theory research: A design framework for novice researchers. SAGE Open Med 2019, 7:2050312118822927.

16. Mierzwinski J, Tyra J, Haber K, Drela M, Sinkiewicz A, Puricelli MD: Pediatric recurrent acute mastoiditis: Risk factors and insights into pathogenesis. Int J Pediatr Otorhinolaryngo/ 2018, 111:142-148.

17. Kirti YK, Yashveer JK: Dilemmas in Current Management of Complicated Chronic Otitis Media. Indian J Otolaryngol Head Neck Surg 2019, 71(2):155-160.

18. McCormick DP, Chonmaitree T, Pittman C, Saeed K, Friedman NR, Uchida T, Baldwin CD: Nonsevere acute otitis media: a clinical trial comparing outcomes of watchful waiting versus immediate antibiotic treatment. Pediatrics 2005, 115(6):1455-1465.

19. Barber $\mathrm{C}$, Ille $\mathrm{S}$, Vergison $\mathrm{A}$, Coates $\mathrm{H}$ : Acute otitis media in young children - what do parents say? Int J Pediatr Otorhinolaryngol 2014, 78(2):300-306.

20. Holl K, Rosenlund M, Giaquinto C, Silfverdal SA, Carmona A, Larcombe J, Garcia-Sicilia J, Fuat A, Munoz ME, Arroba ML et al: The Impact of Childhood Acute Otitis Media on Parental Quality of Life in a Prospective Observational Cohort Study. Clin Drug Investig 2015, 35(10):613-624.

21. Chando S, Young C, Craig JC, Gunasekera H, Tong A: Parental views on otitis media: systematic review of qualitative studies. Eur J Pediatr 2016, 175(10):1295-1305.

22. Hansen MP, Howlett J, Del Mar C, Hoffmann TC: Parents' beliefs and knowledge about the management of acute otitis media: a qualitative study. BMC Fam Pract 2015, 16:82.

23. Meherali S, Campbell A, Hartling L, Scott S: Understanding Parents' Experiences and Information Needs on Pediatric Acute Otitis Media: A Qualitative Study. J Patient Exp 2019, 6(1):53-61.

24. van Uum RT, Venekamp RP, Schilder AGM, Damoiseaux R, Anthierens S: Pain management in acute otitis media: a qualitative study of parents' views and expectations. BMC Fam Pract 2019, 20(1):18.

25. Don DM, Koempel JA, Fisher LM, Wee CP, Osterbauer B: Prospective Evaluation of a Smartphone Otoscope for Home Tympanostomy Tube Surveillance: A Pilot Study. Ann Otol Rhinol Laryngol 2021, 130(2):125-132.

26. Dube E, De Wals P, Gilca V, Boulianne N, Ouakki M, Lavoie F, Bradet R: Burden of acute otitis media on Canadian families. Can Fam Physician 2011, 57(1):60-65.

27. Jonsson $\mathrm{H}$, Haraldsson RH: Parents' perspectives on otitis media and antibiotics. A qualitative study. Scand J Prim Health Care 2002, 20(1):35-39.

\section{Supplementary Files}

This is a list of supplementary files associated with this preprint. Click to download. 
- Table1Demographics.xlsx

- Appendix1 InterviewGuide.docx 